\title{
HispanismeS
}

Revue de la Société des Hispanistes Français

\section{Croyances et résistances de l'écrivain dans Los cuadernos de Rembrandt de José Jiménez Lozano}

Creencias y resistencias del escritor en Los cuadernos de Rembrandt de José Jiménez Lozano

Beliefs and Resistances of the Writer in Los cuadernos de Rembrandt by José Jiménez Lozano

\section{Yannick Llored}

\section{OpenEdition}

\section{Journals}

Édition électronique

URL : https://journals.openedition.org/hispanismes/688

DOI : 10.4000/hispanismes.688

ISSN : 2270-0765

Éditeur

Société des Hispanistes Français

Référence électronique

Yannick Llored, "Croyances et résistances de l'écrivain dans Los cuadernos de Rembrandt de José Jiménez Lozano », Hispanismes [En ligne], 15 | 2020, mis en ligne le 01 juin 2020, consulté le 31 juillet 2021. URL : http://journals.openedition.org/hispanismes/688 ; DOI : https://doi.org/10.4000/ hispanismes.688

Ce document a été généré automatiquement le 31 juillet 2021.

\section{(†) $\ominus$

Les contenus de cette revue sont mis à disposition selon les termes de la Licence Creative Commons Attribution - Pas d'Utilisation Commerciale - Pas de Modification 4.0 International. 


\title{
Croyances et résistances de l'écrivain dans Los cuadernos de Rembrandt de José Jiménez Lozano
}

\author{
Creencias y resistencias del escritor en Los cuadernos de Rembrandt de José \\ Jiménez Lozano \\ Beliefs and Resistances of the Writer in Los cuadernos de Rembrandt by José \\ Jiménez Lozano
}

Yannick Llored

Imposible contar para nada, si se anda por su propio camino vecinal, pero no se trata de contar, se trata de estarse en el propio camino vecinal simplemente.

José Jiménez Lozano, Los cuadernos de Rembrandt.

De moins en moins considéré par les lecteurs comme un genre mineur et confidentiel, le journal personnel - diario en espagnol - d'écrivain constitue une source primordiale pour pénétrer dans l'atelier d'écriture, la conscience critique et la pratique de mise à l'épreuve qui sont dévoilés par un auteur reconnu afin de dialoguer avec soi, tout en s'adressant aux autres souvent connaisseurs de la production littéraire de ce dernier. Dans ce dialogue implicite, il s'agit pour l'écrivain d'exprimer d'abord des points de vue - en relation indirecte avec les principes de différents ordres dont est porteuse son œuvre littéraire - concernant des problématiques collectives tant historico-culturelles qu'éthico-politiques, voire sociétales, mais aussi d'inscrire la singularité d'une prise de position critique au sein de laquelle peuvent s'allier la réflexion lucide et l'antagonisme de valeurs conflictuelles reliées à un champ intellectuel déterminé.

Depuis le milieu du XX $\mathrm{XX}^{\mathrm{e}}$ siècle et même avant - pensons notamment à Miguel de Unamuno, à Azorín, et, de façon différente, à Antonio Machado -, la littérature espagnole contemporaine cultive assez largement le genre du diario dont les critères génériques demeurent fluctuants et marqués par une certaine hybridation, située en 
particulier entre l'essai et l'autobiographie ${ }^{1}$. Le diario littéraire développe un lien particulier entre la connaissance, plus ou moins intime, de l'auteur et l'approfondissement fragmentaire, à partir de différentes formes de subjectivité, de sa pensée, de ses affects, du retour réflexif sur les expériences vécues et de la réalité complexe du monde qui l'entoure. On peut dire que chaque écrivain significatif - à travers, entre autres éléments, la reconnaissance et le capital symbolique acquis par son œuvre - conçoit ses propres caractéristiques du diario. Celui-ci demeure principalement centré sur l'introspection du sujet en configurant les formes, les propriétés et les contours assez mouvants d'un genre qui se redéfinit en rapport étroit avec la poétique d'une écriture singulière ${ }^{2}$ et la figure souvent diverse de soi qui s'y façonne. Ainsi, par exemple, le Diario de un poeta seriamente enfermo (1974) de Jaime Gil de Biedma, La gallina ciega (1971) de Max Aub et les nombreux volumes du Salón de los pasos perdidos (1990-2018) de Andrés Trapiello sont bien des diarios - ou journaux personnels d'écrivains -, mais leurs caractéristiques de composition, les différentes approches de l'intériorité du narrateur, leurs conceptions esthétiques et visées critiques confrontent les lecteurs à des univers littéraires, des procédés stylistiques et des régimes textuels très différents. C'est d'ailleurs Andrés Trapiello qui, dans un compte rendu de Los cuadernos de letra pequeña (2003) de José Jiménez Lozano (1930-2020), essaie de préciser certaines caractéristiques formelles de la pratique d'écriture du diario chez ce dernier :

Y así entramos ya en lo que son estos diarios. Como en todos los suyos no hay entradas por días, sino trozos, fragmentos, relucencias, que diría él. La mayor parte de esos fragmentos se los arranca una noticia del periódico, una inquietud espiritual, las trampas de la cultura moderna, el descreimiento contemporáneo, un libro. Raramente su privacidad, a salvo ${ }^{3}$.

3 En effet, à la différence des journaux intimes centrés sur le «moi » et l'intériorité de l'écrivain, qui est sondée à la lumière des variations d'une approche introspective, les diarios de Jiménez Lozano constituent plutôt des cahiers de notes où l'auteur établit, dans sa quotidienneté, un dialogue critique avec une réalité politique, socioculturelle et même spirituelle, face à laquelle il puise l'acuité d'un regard contestataire et d'une conscience interpellatrice portés sur les dérives du monde contemporain. En ce sens, l'écriture des journaux - ou cahiers de notes - de Jiménez Lozano s'inscrit plus exactement dans le genre du dietario ${ }^{4}$. Car la figure et la voix de l'écrivain sont comme tournées vers le dehors inhérent à une réalité commune et, tout en développant leurs liens avec l'expérience d'une quotidienneté, tendent à éclairer les cheminements d'une espèce d'aventure morale au sein de laquelle l'enjeu consiste aussi à pouvoir reconnaître sa place, son lieu et son être dans et face au monde ${ }^{5}$.

4 Les carnets de bord des lectures, des réflexions critiques et des pensées, soigneusement sélectionnés et réunis au fil des années, se transforment ainsi en l'espace protéiforme non pas d'un examen de conscience, mais bien plutôt d'un affrontement moral. Ce dernier s'érige, par ses écarts de vision, ses différences discordantes et son intériorité paradoxale, face aux évolutions et à l'état d'une société ainsi que de son humanité. De fait, depuis le milieu des années 1980, avec la publication de Los tres cuadernos rojos, qui contribua à consolider l'intérêt pour le dietario dans la littérature espagnole contemporaine, jusqu'à la fin de sa vie, marquée, entre autres, par la parution de Cavilaciones y melancolías (2018), Jiménez Lozano ne cessa de faire connaître, en parallèle à son œuvre importante d'auteur de récits brefs - cuentos en espagnol -, de romancier, de poète, d'essayiste et de collaborateur régulier de la presse nationale, ses cahiers de 
notes personnelles ${ }^{6}$. Au sein de ceux-ci, les figures, les voix et les activités d'écriture de l'écrivain interagissent, de manière diffuse, afin de donner à voir et à comprendre ce qui, pour lui, s'avère primordial et on ne peut plus inquiétant dans le délitement des formes de vie, du dire, de la pensée et, finalement, d'exister au monde. C'est l'exigence d'une comparution vis-à-vis de ces formes et, donc, des pratiques s'y rattachant, qui renouvelle en permanence un acte de résistance propre aux convictions clairement affirmées de Jiménez Lozano, lequel est assez amplement reconnu pour son attachement à la foi chrétienne, son esthétique littéraire parfois qualifiée de "prémoderne" et son positionnement critique plutôt traditionnel lié à un conservatisme éclairé soucieux de sa précieuse indépendance.

5 Ainsi, situés au croisement du journal personnel, de l'essai, de la chronique politicoculturelle, du témoignage subjectif et même de la création littéraire - on relève plusieurs poèmes dans Los cuadernos de Rembrandt (2010) -, les dietarios de Jiménez Lozano, ponctués par les incessantes lectures, par le passage cyclique des saisons et les événements souvent tragiques d'une actualité, offrent la possibilité de pénétrer la nature singulière d'une expérience littéraire qui se veut, du côté de sa visée de réception, comme accompagnatrice des préoccupations, des doutes et perplexités des lecteurs ${ }^{7}$. Au cœur de cette expérience littéraire sont interrogés les différents univers et modes d'être de l'existence du sujet - à savoir, le soi observateur-témoin et surtout les autres observés -, qui se trouve confronté aux processus sociohistoriques d'un certain phénomène de dé-civilisation indissociable de la perte de valeurs que l'on peut qualifier d'interhumaines. Cette perte transparait dans les trois thématiques centrales qui parcourent Los cuadernos de Rembrandt (2010) : il s'agit des liens aux traditions et aux héritages culturels inséparables de la notion-clé de transmission - d'où les considérations sur l'éducation scolaire, sur l'Europe contemporaine et la religion --, des réflexions partisanes sur les évolutions d'ordre sociétal souvent liées aux discours socio-politiques dominants - telles que les questions de l'avortement et de l'euthanasie, par exemple, mais aussi, sur un autre plan, les débats autour de la mémoire historique et, enfin, la défense d'une certaine conception de la littérature inhérente à un attachement intime à une écriture où le langage naturel doit toujours viser à faire éclore une vérité dont la portée révélatrice s'ouvre sur une dimension transcendante ${ }^{8}$.

6 À partir de ces prémisses d'analyse, nous aborderons le dietario intitulé Los cuadernos de Rembrandt - car la couverture des cahiers de notes dont est issu l'ouvrage présentait des gravures du maître hollandais admiré par Jiménez Lozano - en fonction d'une double approche. Il s'agit, d'une part, de mettre en lumière la manière dont l'écriture déploie une poétique qui comprend par intermittences une conception singulière de l'art de la narration, des formes d'expression littéraire et de la figure de l'écrivain, et, de l'autre, d'interroger les prises de position de ce dernier au regard de ses principes d'ordre éthique et d'une conscience morale inséparables d'un humanisme critique - supposé dépassé -, ainsi que de certaines considérations spirituelles axées sur une ontologie de l'existence humaine.

7 À l'écart de l'adhésion ${ }^{9}$ ou des réserves, plus ou moins appuyées, que tout lecteur peut éprouver à l'égard des observations et des avis exposés par l'écrivain de façon assez insistante au fil des pages de son dietario, un principe structurant se dégage du positionnement de ce qui est formulé par le sujet d'écriture dans Los cuadernos de Rembrandt. Ce principe réside dans l'homologie évidente entre les prises de position de Jiménez Lozano - souvent situées à contre-courant des tendances et des formes de 
pensée dominantes - et la position assez périphérique qu'occupe l'auteur au sein du champ littéraire et intellectuel espagnols, et ce, malgré la reconnaissance institutionnelle dont il a pu bénéficier ${ }^{10}$. Par conséquent, c'est une espèce de discordance paradoxale que cultive l'écrivain comme représentant reconnu des valeurs traditionnelles de la culture de l'identité castillane - ouverte aussi sur d'autres cultures constitutives de cette identité plurielle, en particulier le judaïsme et le mudejarismo - et, en même temps, comme critique acerbe de tout ce qui établit un rapport de pouvoir, de domination et d'imposition entre le sujet et sa manière de penser et d'agir. Jiménez Lozano montre précisément comment ce rapport contribue à étouffer la radicalité lucide d'une voix singulière et souvent dénonciatrice des stratégies de capitalisation et de reconnaissance intéressées, qui s'avèrent prépondérantes au sein des règles d'un milieu - et, finalement, d'une sorte de marché - littéraire, culturel et politique.

Dès lors, une tension ne cesse de s'affirmer au sein des propos et des perspectives d'énonciation de la voix narrative dans Los cuadernos de Rembrandt, dont les notes réunies dans ce dietario s'échelonnent de l'année 2005 jusqu'à 2008. Cette tension se déploie entre, d'une part, la revendication du fameux « moi haïssable " pascalien et, de l'autre, la nécessité non moins ferme de la quête d'authenticité du sujet, de son intériorité et de son lien essentiel au monde. Cette tension est de même centrale dans l'autoportrait en creux qui se trouve ébauché, par touches à la fois successives et fragmentaires, dans l'ouvrage apte à accueillir une multiplicité de formes d'écriture telle une constellation gravitant sur elle-même et composée des figures tutélaires, des voix, des silences et questionnements parcourant assez largement l'œuvre de Jiménez Lozano depuis le milieu des années 1970. Ainsi, en rejetant à travers l'ironie acerbe l'étiquette de " escritor católico ${ }^{11}$, qui vise à enfermer ce dernier dans sa condition de croyant prétendument à l'écart des attentes du public, l'auteur tend à expliciter sa perception intérieure de l'écriture en se qualifiant lui-même de « escribidor » afin de sonder la nature intime des liens au langage littéraire :

[...] pienso que el lenguaje se le regala a uno con la historia que está contando. 0 por lo menos no sabría yo qué hacer para limpiar las palabras antes de ponerme a escribir. No creo que el escritor tenga poder sobre ellas; pienso que todo está en acertar a ponerlas de manera que con ellas nombre, que es lo que tiene que hacer una palabra: levantar realidad y vida. Y lo certísimo es que, si se acierta con las palabras que nombran y su ordenación, éstas contagian, con su candor primero, a quien lee y a quien escribe ${ }^{12}$.

9 Fidèle aux conceptions esthétiques de ses figures tutélaires que sont, pour Jiménez Lozano, les Messieurs de Port-Royal - notamment, Pierre Nicole et, de manière plus profonde, Blaise Pascal -, qui rejettent toute rhétorique factice et tout artifice trompeur dans l'écriture afin que celle-ci exprime une vérité et une beauté propres à révéler l'authenticité naturelle du sens des $\operatorname{mots}^{13}$, l'auteur de Los cuadernos de Rembrandt unit les principes-clés de son art de la narration et de l'écriture à une dimension morale s'ouvrant sur un horizon d'interrogation d'ordre éthique.

De même, cet art se nourrit principalement de la tradition du récit biblique et, en particulier, de son héritage hébraïque. En lien étroit et permanent avec cette tradition, Jiménez Lozano se reconnaît avant tout comme " escribidor ${ }^{14}$ pour signifier qu'il se situe aux antipodes de la figure du créateur-démiurge en recherche permanente de réinvention et de transformation de l'art littéraire.

11 Le nom de « escribidor », tel qu'il est employé par Jiménez Lozano, rend compte de sa conception et de son appréhension de l'écriture en littérature considérée non pas 
comme un acte de création originale auto-suffisant, mais bien plutôt comme celui d'une réception intériorisée, d'un accueil transfiguré et d'une écoute intérieure de faits, de personnages, de lectures ainsi que d'expériences vécues et remémorées. Ainsi, l'écrivain soucieux de se soustraire à son « moi » - néanmoins bien présent, comme on l'observera, dans certaines de ses prises de position concernant des problématiques collectives - accueille dans son intériorité profonde des représentations, des figures et des voix qui s'imposent à lui en surgissant de son for intérieur, de sa mémoire et sa conscience créatrice. C'est au cœur de cette expérience éprouvante et toujours incomplète ${ }^{15} \mathrm{~d}^{\prime}$ une matière dense et multiple qui lui advient que Jiménez Lozano fait de son écriture un lieu essentiel d'écoute, de dépouillement et de transfiguration de la parole et la présence de l'autre et des autres souvent rendus invisibles - ou insignifiants - par les discours dominants et le progrès de la supposée modernité. Le " escribidor » est alors une sorte d'arpenteur-(re)découvreur en écriture qui demeure rivé aux héritages culturels intériorisés, ainsi qu'à la parole et aux voix des autres ${ }^{16}$.

Dans cette optique, les principes esthétiques propres à l'écriture de Jiménez Lozano et, de façon corrélative, aux traits définitoires de la tâche de "escribidor " amènent l'auteur à faire éclore l'avènement d'une réalité spirituelle ${ }^{17}$ au sein du langage littéraire, mais également à redonner sens à des notions éthico-religieuses, telles que celles de compassion, de joie intérieure et d'espérance. Celles-ci permettent à l'écrivain d'essayer de nommer le noyau de l'intériorité du sujet afin de résister et de lutter contre la cruauté, la misère et l'abandon qui minent l'humanité d'un monde se trouvant écartée de l'interrogation fondamentale et de l'espace essentiel de questionnement d'ordre éthique relatifs au sens de la vie.

13 Ce n'est donc aucunement fortuit si les liens ramifiés entre conception esthétique de l'écriture et interrogation éthique sur l'existence humaine conduisent Jiménez Lozano à dénoncer l'instrumentalisation, voire le dépérissement, de toute morale authentique dans une large partie de la production littéraire contemporaine comme pour mieux signifier a contrario ce qui, selon lui, doit être encore et toujours recherché dans l'écriture et la lecture d'une œuvre littéraire :

[...] una obra literaria sin moral, que ahora quiere decir sin ideología de progreso contra hipocresías y maldades, reales o supuestas, no cuenta en literatura. Es la moral, ciertamente, la que tiene gancho y embellece la obra y al autor, y seduce al público. La literatura en el mundo moderno nunca ha ido muy lejos por sí sola, y la crítica de la industria cultural -exactamente como los pregoneros de las representaciones teatrales que se hacían en las aldeas cuando yo era niño decían que aquéllas eran "altamente morales"- dice ahora, para consagrar a pintores o escritores, que son subversivos e inconformistas y liberadores, pero sobre todo "éticos" e incluso "místicos", algo que no quiere decir nada, pero que se vende muy bien $^{18}$.

14 En raillant l'hétéronomie servile d'une production artistique qui se complait dans une supposée esthétique transgressive et émancipatrice ${ }^{19}$, laquelle devient, en fait, un lieu commun de la doxa des tendances dominantes au sein des règles du marché de l'industrie culturelle, Jiménez Lozano ne réhabilite pas une espèce d'élitisme littéraire réservé aux lecteurs "élus ». En revanche, il réaffirme à travers l'opposition et le renversement argumentatif la nécessité première de l'écriture de faire écho à la radicalité nue de la vie, à sa fragilité et vulnérabilité, et ce, en éclairant par la traversée des ténèbres et des lueurs d'espoir les questionnements ultimes d'une existence - son champ des possibles et ses limites sur le plan éthique - grâce aussi à l'élaboration des 
conditions d'une lecture méditative, révélatrice et, finalement, réparatrice des blessures d'une expérience vitale et de sa mémoire parfois meurtrie.

La figure du « escribidor » - arpenteur d'épreuves en quête de vérité et de dévoilement intérieur grâce à une écriture ouverte aux voix, aux présences et silences des autres s'allie dans le dietario à celle de l'intellectuel parfois moralisateur, qui ne cesse d'interpeller indirectement la conscience des lecteurs, mais aussi aux paroles du sujet poétique énonçant les pâles vibrations intérieures d'une ultime lueur d'espoir déclinante :

Entre la neblina de la fina lluvia,

sol tornasolado que ilumina

el oro mate de los árboles ya heridos

por el cuchillo del otoño.

Todavía un último verdor exasperado,

como siempre lo es la última esperanza ${ }^{20}$.

L'atmosphère semi-lumineuse et la variation des nuances chromatiques, ainsi que celle des impressions visuelles - influencées par la peinture d'un Georges de la Tour ou d'un Honthorst - ancrent subtilement le regard et la voix de l'écrivain-poète dans une terre et une nature. Mais ce regard et cette voix comprennent aussi une transfiguration subtile de ce qui est dit et montré afin de déployer en filigrane la densité d'une réalitéexpérience spirituelle. Dès lors, située au seuil de la fatalité d'un destin voué à la disparition et de l'élan irrépressible vers un au-delà, la « última esperanza » devient ce reste-résidu vital qui continue nécessairement à se faire attendre et entendre comme seul espoir d'une fragile force de vie et d'humanité, au sein de laquelle le sujet peut se reconnaître à lui-même et à ce qui l'attache au monde dans sa réelle présence. C'est une autre variation sémantique de la notion-clé de " esperanza » qui se trouve signifiée plus loin dans le dietario en passant de la dimension spirituelle à la mise en question morale, en lien avec un état avilissant du monde et des hommes :

Todo parece como si la compasión, al igual que la esperanza, fuera cierta espontaneidad natural en algunas personas y en otras una especie de herencia cultural que en principio resultan sabrosas, pero que luego se tornan insípidas. Enseguida se nos convierte en ridícula u odiosa esa compasión, y volvemos a "la realidad", y entonces pensamos que, bien echadas las cuentas y sopesadas todas las circunstancias, los verdugos no resultan, al fin y al cabo, tan horribles y seguramente estuvieron justificados en lo que hicieron. Al menor descuido, los buenos sentimientos se convierten en asesinatos más o menos simbólicos ${ }^{21}$.

L'accusation portée sur le dépérissement moral, qui affecte les individus et leurs critères de jugement, sous-tend le constat relatif au délitement des rapports au passé historique et aux valeurs culturelles quant à leur pouvoir de signification et de transmission pour continuer à interroger notre humanité. De la sorte, sur un plan corrélatif, si l'œuvre de la philosophe Simone Weil peut devenir une lecture à la mode dans un cercle restreint d'intellectuels médiatiques, en se voyant comme dépouillée de sa radicalité critique, ou si celle du poète Paul Celan peut être parfois interprétée de manière tendancieuse, comme teintée d'une esthétique kitsh - alors même qu'elle est un témoignage poétique de grande envergure de l'expérience de l'extermination et de la langue des bourreaux nazis -, c'est parce que les relativismes et révisionnismes de la prétendue postmodernité s'accompagnent de pratiques d'idéologisation et de dénégation qui sont dénoncées, ici, par Jiménez Lozano ${ }^{22}$.

La compassion n'est plus alors un des repères intérieurs du sujet moral, un de ses principes essentiels de compréhension et de solidarité à l'égard de la souffrance et la 
mémoire des victimes - et, donc, de la réalité extrême éprouvée par l'autre -, mais elle devient une espèce d'instrument des préjugés partagés, des opinions préconçues et des arrangements subordonnés à l'air du temps et adoptés par une partie de la société. Celleci peut ainsi se prétendre comme libérée du passé parfois à l'aide de bons sentiments empreints des nouveaux dogmes - souvent politiquement corrects - d'une époque donnée.

En fonction d'un point de vue bien distinct et quelque peu partisan de la part de l'écrivain, il convient de relever comment ce dernier montre un positionnement plutôt paradoxal concernant la mémoire historique relative à la Guerre civile espagnole (1936-1939). À l'écart de la mise en avant d'une volonté de justice et d'une revendication de la mémoire des victimes du conflit fratricide, Jiménez Lozano dénonce plutôt ce qu'on appelle en Espagne le "guerracivilismo", à savoir la résurgence politisée des profondes déchirures du passé historique dans l'objectif de revendiquer la pleine reconnaissance sociale et politique des victimes du franquisme. De la sorte, c'est l'utilisation d'une mémoire instrumentalisée qui est rejetée par l'écrivain, lequel ne cessa d'ausculter dans certains de ses essais ${ }^{23}$ la question du « caínismo » comme source de fractures très anciennes et bien idéologisées au sein de la société et de l'Histoire espagnoles :

Se están abriendo fosas comunes del tiempo de la guerra civil española de 1936-1939 -aunque solamente las de uno de los lados combatientes-, y un juez erigido en Juez de los Vivos y Muertos como Radamante se presenta haciendo una justicia escatológica e histórica a la vez, un Juicio Último inmanente, pero que dividiría los tiempos. [...] Más valdría que maceráramos en nuestro corazón toda huella de odio, y nos avergonzáramos de nosotros mismos, porque ya es tiempo de que nuestra España se libere, de una vez por todas, de memorias y castas malditas, "de los hunos y los hotros", como escribía tan doloridamente don Miguel de Unamuno. Nunca hubo dos Españas, sólo hubo siempre una sola, triste, y desgarrada España ${ }^{24}$.

20 Il ne s'agit pas, ici, pour l'écrivain, de réaffirmer son attachement indéfectible à la memoria passionis des vaincus et victimes des processus historiques, mais de souligner dans une certaine affinité avec les positions de l'historien-philologue Américo Castro ${ }^{25}$ - les ravages multiséculaires provoqués en Espagne par le "caínismo » et, donc, la reproduction des conflits procédant des luttes acharnées antre différentes idéologies, croyances et positions politiques. Pour Jiménez Lozano, la mémoire historique nécessairement indissociable d'une dimension politique - ne peut que raviver les incompréhensions et déchirures du passé national. Ce sont, par conséquent, les limites de cette mémoire qui sont exposées par l'écrivain qui ne tend pas à éclairer comment celle-ci peut aussi redéfinir les rapports que chacun construit avec le passé et qu'elle est de même source de connaissances et, dans une certaine mesure, réparatrice des injustices et dénis transmis par le passé historique; ces derniers sont, de fait, fréquemment refoulés par une conscience collective fracturée. Replié sur la partialité de son point de vue légitime et assez partagé ${ }^{26}$ en Espagne, Jiménez Lozano privilégie au fond le silence du recueillement évocateur et, à la fois, protecteur de la mémoire de toutes les victimes qui prévaut dans la reconnaissance de leur souffrance, et ce, en n'abordant pas la signification politique des crimes subis par ces victimes, le sens de leur combat et ce qu'il peut transmettre aux générations futures.

21 Il importe de relever que les notes, les réflexions et textes brefs constitutifs de Los cuadernos de Rembrandt correspondent aux années 2005 à 2008 au cours desquelles le gouvernement socialiste de José Luis Rodríguez Zapatero essaya de mener à bien une 
série de réformes sociétales - souvent qualifiées de "progressistes" avec les sousentendus quelque peu péjoratifs -, qui font l'objet des critiques parfois virulentes et dotées d'un certain trait accusé de Jiménez Lozano. Ainsi, l'écrivain réputé conservateur adopte apparemment une posture en surplomb qui ne manque pas de dénoncer la corruption d'un discours politique apte à tout dire et son contraire, et qui n'est qu'un instrument habile pour défendre les intérêts et pouvoirs des dominants. De même, les annotations désabusées sur le système éducatif soulignent la perte de vraies valeurs de transmission culturelle à cause d'une sorte de pédagogisme du nivellement postulant naïvement la réussite de tous et aspirant à une école détachée de l'héritage des traditions vécues dans le passé, ainsi que de leurs legs identitaires ${ }^{27}$. D'autre part, les brefs commentaires sur le terrorisme islamiste qui, comme on le sait, frappa très durement Madrid en mars 2004 et, de façon moins dévastatrice, les années suivantes ${ }^{28}$, donnent lieu dans le dietario à une critique acerbe concernant non seulement une action politique qui peut manipuler, par intérêts stratégiques, les masses, mais aussi une Union européenne ${ }^{29}$ pusillanime en manque de vrai courage politique afin de lutter contre ses agresseurs et reniant ses racines chrétiennes et leurs héritages culturels. Dans cette perspective, Jiménez Lozano soutient que les sociétés européennes paraissent comme soumises à la peur, elles préfèrent pactiser secrètement avec leurs ennemis au lieu de les affronter véritablement au plan politique et idéologique en amenant, finalement, la grande masse des citoyens à se soumettre à une situation au sein de laquelle prédominent les intérêts particuliers, les relativismes de tous bords et les tristes passions du monde: "Todos debemos experimentar el infierno en este mundo, para convencernos de la bondad de nuestro sometimiento $»^{30}$.

Cette dénonciation de la soumission de l'individu aux intérêts du pouvoir politique, à la manipulation de l'arme qu'est la peur et au reniement des valeurs historiques d'une identité collective s'allie, chez l'écrivain, à sa critique du multiculturalisme et de la notion diluée par les préjugés de tolérance. Cette critique contraste, là aussi, assez nettement avec certaines de ses positions intellectuelles sur Al-Andalus et la culture mudéjare dans une filiation sans équivoque aux thèses d'Américo Castro sur l'Espagne des trois religions ${ }^{31}$. En ce sens, le fameux " politiquement correct ", les points de vue officiels préconçus par les consensus de différents types et les croyances collectives où règne l'influence des opinions prédéterminées se chargent, selon Jiménez Lozano, de faire admettre ce qui peut être reçu et transmis aux autres en accord avec les critères de la doxa admise par une société donnée à une époque précise.

De manière corrélative, les questions complexes de l'avortement et de l'euthanasie sont abordées de façon assez récurrente dans le dietario en montrant le positionnement très ferme de l'intellectuel catholique qui s'érige à nouveau contre la réification de l'être humain, sa déshumanisation et le mépris, plus ou moins implicite, projeté sur la vie des plus fragiles et vulnérables:

Esta otra peste se sabe muy bien de dónde viene; aparece en el darwinismo filosófico del XIX, y estuvo en la base de las prácticas del higienismo hitleriano; y en Núremberg se llamó Science in Beemoth con el nombre de la terrible ballena bíblica, símbolo del Mal, y ahora resulta que se llaman "derechos". Y son derecho a impedir el nacimiento de otro ser humano que -para eliminarlo- se denomina al efecto "desecho sanitario"; o, en el caso de la eutanasia de Estado, derecho a anticipar una "muerte digna" a alguien de quien se decide que no tiene ya "calidad de vida" o ha vivido ya bastante. Cuestiones de contabilidad de granja en suma: el control de un "plus" de nacimientos no queridos ni útiles, y de un "plus" de ganado ya no productivo o rentable de otro modo ${ }^{32}$ 
dè du propos quelque peu provocateur - à travers, par exemple, le rapprochement à l'hygiénisme hitlérien -, la charge accusatrice de Jiménez Lozano établit indirectement un contraste paradoxal, ici aussi, avec sa défense de l'autonomie du sujet dans l'affirmation de ses choix et de son libre arbitre. Car les questions difficiles de bioéthique, telles que celles de l'avortement et de l'euthanasie, ne s'ancrent pas essentiellement dans une sorte d'administration étatique des corps, mais bien plutôt dans la reconnaissance des droits d'un sujet qui peut décider de contrôler, dans une certaine mesure, son destin corporel et, donc, biologique en affrontant nécessairement des questionnements fondamentaux. Ce processus d'individuation du destin biologique du sujet, encadré par l'État et ses critères juridiques, n'est pas directement subordonné dans un pays démocratique, tels que la France ou l'Espagne, à des phénomènes de contrôle social strict ni à l'intériorisation de normes axiologiques différenciant ceux qu'on laisse vivre et ceux qu'on laisse mourir. Jiménez Lozano noircit, donc, le trait et semble manquer de nuances pour défendre sa cause essentielle pour la vie inséparable de la croyance chrétienne, et ce, en détournant quelque peu la valeur et le sens du droit du sujet de s'approprier son corps sans pour autant ne point respecter la vie même si cette dernière est considérée à l'écart de sa présumée sacralité ainsi que d'une perspective théologique.

Los cuadernos de Rembrandt s'avère représentatif de l'écriture multiple des dietarios de Jiménez Lozano. Ce dernier offre à ses lecteurs des approches et des perspectives critiques dissonantes - et parfois non détachées de la foi - afin de s'interroger sur soi et sur une réalité contemporaine où l'humain, le sens de la vie et les questionnements d'ordre éthique sur nos rapports à l'existence, se trouvent, selon l'écrivain, de plus en plus réduits par une raison instrumentale et par les relativismes prédéterminés, tant cultuels que moraux, détournant le sujet d'une véritable quête de vérité pas seulement intérieure. Néanmoins, les certitudes inhérentes à la foi de l'écrivain - sa confiance en la vraie croyance - l'amène à des prises de position unilatérales qui peuvent heurter le lecteur en opérant un décentrement assumé et radical par rapport aux discours conventionnels et socialement acceptés. Loin d'adopter une tonalité confessionnelle, Jiménez Lozano crée en réalité un foyer de résistance active au moyen de l'écriture du dietario, non seulement pour susciter chez son lecteur le fait d'appréhender ce qu'il y a de plus grand que lui - ce qui le passe et dépasse en lien parfois avec une transcendance -, mais aussi pour essayer de redonner sens à ses actes, ses manières de penser, de parler, de comprendre le monde environnant et les autres souvent rendus invisibles. La modulation parfois moralisatrice, liée à des positionnements conservateurs, qu'adopte à certains moments la voix de l'écrivain témoigne en partie de la non-contemporanéité de sa pensée face aux évolutions sociétales. Néanmoins, cette pensée partisane vise aussi, en éclairant des formes d'impensées et en évoquant la permanence primordiale des héritages culturels et spirituels, à favoriser un débat qui accorde une centralité à la reconnaissance de la liberté du sujet - à son intégrité morale - et à l'écoute d'une parole autre et discordante souvent rendue muette par les discours sociaux et médiatico-politiques dominants, au sein desquels la notion acceptée de progrès peut parfois porter atteinte à la vraie liberté du sujet. En ce sens, c'est bien une interpellation implicite qu'adresse avec insistance José Jiménez Lozano à son lecteur. L'écrivain réaffirme ainsi la souveraineté de ce que la personne humaine - au-delà de ses émotions, ses sentiments et affects - possède de plus profond et singulier dans ses attaches et ses liens constitutifs de sa présence authentique au monde, et ce, pour 
continuer à sonder le pouvoir de révélation de la réalité de ce monde, mais aussi nécessairement pour préserver sa force de révélation de soi grâce à l'intensité d'une joie intérieure source originelle d'humanité et de vérité33 toujours à ressaisir :

Uno de los personajes de Sade, un príncipe, dice que los pueblos deben saber que hay un infierno inmanente y que hay que hacérselo sentir a esos pueblos para que sean bien mandibles. $Y$ tales parecen ser nuestros señores hoy. Hasta se ocupan de nuestras diversiones, que deben ser pedagógicas y rentables. Nadie debería guardar la alegría, su alegría propia, porque sería como una bomba que se llevaría todos los estaribeles por delante. $Y$ lo es $^{34}$.

\section{BIBLIOGRAPHIE}

Anna CABALLÉ, « Entrevista a Miguel Sánchez-Ostiz », Boletín de la Unidad de Estudios Biográficos, n 1 (1996), p. 77-81.

Ana CALVo ReVILLA, « Retórica política, cultura y poder. Disputas y controversias en Los cuadernos de Rembrandt de José Jiménez Lozano ", Emilio del Río Sanz, María del Carmen Ruiz de la Cierva et Tomás Albaladejo (éd.), Retórica y Política. Los discursos de la construcción de la sociedad, Logroño, Instituto de Estudios Riojanos, 2012, p. 573-589.

Américo CASTRO y José JIMÉNEZ LOZANO, Correspondencia 1967-1972, Introducción, edición crítica y notas de Guadalupe Arbona y Santiago López-Ríos, Madrid, Trotta, 2020.

Danielle CORRADO, « Le journal intime en Espagne : indifférence ou différences ? », Cahiers de l'Echinox, vol. 16 (2009), p. 294-306.

Hans Georg GADAMER, L'Art de comprendre. Écrits II. Herméneutique et champ de l'expérience, Paris, Aubier, 1991.

Jordi GRACIA, « La virtud del intruso, el dietario de escritor (segunda parte, 2000-2017) », Cuadernos Hispanoamericanos, $\mathrm{n}^{\circ} 811$ (2018), p. 36-53.

José JIMÉNEZ LOZANO, El mudejarillo, Barcelona, Anthropos, 1992.

José JIMÉNEZ LOZANo, Sobre Judíos, moriscos y conversos. Convivencia y ruptura de las tres castas, Valladolid, Ámbito, 2002.

José JIMÉNEZ LOZANo, Guía espiritual de Castilla, Valladolid, Ámbito, 2004.

José JIMÉNEZ LOZANO, Los cementerios civiles y la heterodoxia española, Barcelona, Seix Barral, 2008.

José JIMÉNEZ LOZANO, Los cuadernos de Rembrandt, Valencia, Pre-Textos, 2010.

José JIMÉnEZ LOZANo, Siete parlamentos en voz baja, Almería, Confluencias, 2015, p. 137-149.

Yannick LLORED, « La pensée de Blaise Pascal et de Port-Royal dans l'écriture de José Jiménez Lozano ", Nicole Fourtané et Michèle Guiraud (dir.), Emprunts et transferts culturels dans le monde luso-hispanophone : réalités et représentations, Nancy, Presses Universitaires de Nancy, 2011, p. 189-205. 
Álvaro LUQUE AMO, «El diario personal en la literatura: teoría del diario literario», Castilla. Estudios de Literatura, vol. 7 (2016), p. 273-306.

Álvaro LUQUE AMO et Michel BRAUD, « El establecimiento del diario personal en el sistema literario: el diario literario en Francia y España », Revista de Literatura, n 164, vol. LXXXII (2020), p. 347-373.

Reyes MATE, Tratado de la injusticia, Barcelona, Anthropos, 2011.

Rafael SÁNCHEZ FERLOSIO, La hija de la guerra y la madre de la patria, Barcelona, Destino, 2002.

Matthieu SERGIER et Sonja VANDERLINDEN, « Le journal d'écrivain. Les libertés génériques d'une pratique d'écriture ", Interférences littéraires / Literaire interferenties, n 8, 2012, p. 7-14.

Andrés TRAPIELLO, « Palabras mayores », Babelia-El País, numéro du 8/03/2003, p. 5.

\section{NOTES}

1. Les formes d'évolution et d'établissement du diario dans le champ littéraire espagnol et français sont étudiées, à partir d'une perspective historique, dans l'article d'Álvaro LUQUE AMO et Michel BRAUD, «El establecimiento del diario personal en el sistema literario: el diario literario en Francia y España », Revista de Literatura, n 164, vol. LXXXII (2020), p. 347-373.

2. Matthieu SERGIER et Sonja VANDERLINDEN, « Le journal d'écrivain. Les libertés génériques d'une pratique d'écriture », Interférences littéraires / Literaire interferenties, $n^{\circ} 8,2012$, p. 12-13 : «[...] le journal d'écrivain semble disposer d'une force de déviance qui lui permet de parasiter d'autres genres, de muter au gré des courants esthétiques, de se métisser en fonction des poétiques d'auteurs et des circonstances spatio-temporelles. [...] Le genre se prête par excellence à une pratique à l'écart du discours officiel, se complaisant dans des situations radicalement paratopiques ".

3. Andrés TRAPIELLO, «Palabras mayores», Babelia-El País, numéro du 8/03/2003, p. 5.

4. Comme le précise Danielle CORRADO, «Le journal intime en Espagne: indifférence ou différences? », Cahiers de l'Echinox, vol. 16 (2009), p. 295 : «La pratique du dietario se tient au confluent du journal intime et du journalisme culturel et littéraire très présent dans la presse espagnole depuis le XIX $\mathrm{X}^{\mathrm{e}}$ siècle ; les "dietaristes" sont de fait souvent écrivains et journalistes. [...] [L]e dietario dessine un moi littéraire qui se construit jour après jour, année après année ».

5. À propos de certaines caractéristiques du diario d'écrivain, on peut aussi lire l'article de Álvaro LUQUE AMO, «El diario personal en la literatura: teoría del diario literario », Castilla. Estudios de Literatura, vol. 7 (2016), p. 295 et p. 300 : « [...] el diario estaría protagonizado por el punto de vista del autor, mientras que en el dietario el autor se difumina, y su perspectiva es solo una excusa para hablar de los temas más diversos. [...] [E]l diario personal sería más bien aquel texto en el que el yo se espacia en un terreno íntimo o privado, construyéndose hacia el interior, en contraposición a un terreno más cercano a lo público, en donde se desarrollaría el dietario. [...] Como aclara Caballé, la palabra dietario procede etimológicamente de diaeta (no así de diarium) que significa "manera de vivir" o "régimen de vida", y en castellano y en catalán se ha venido aplicando a los libros de contabilidad, es decir a un registro más formal y trascendente de la actividad literaria ».

6. Signalons qu'après le décès de l'écrivain, en mars 2020, le dernier dietario de J. Jiménez Lozano a été publié en Espagne sous le titre de Evocaciones y presencias (Madrid, Confluencias, 2021).

7. Concernant le genre du dietario, on peut relever les observations de l'écrivain Miguel SánchezOstiz dans Anna CABALlé, "Entrevista a Miguel Sánchez-Ostiz », Boletín de la Unidad de Estudios Biográficos, $\mathrm{n}^{\circ} 1$ (1996), p. 77 : «El dietario, a mi modo de ver, es un cajón de sastre, una novela desordenada, un diario de a bordo, en el que cabe desde el ejercicio introspectivo hasta el 
memorialístico, pasando por el de testigo de mirada oblicua o marginal de su época, las opiniones más o menos contundentes, sus estampas más o menos sentimentales de cosas vistas, de lecturas, de humores encontrados incluso [...]».

8. Ces thématiques-clefs assez récurrentes témoignent bien du fait que nous sommes face à un dietario dans Los cuadernos de Rembrandt et qu'il est impératif de ne pas le confondre avec le diario, car comme le signale Jordi GRACIA, «La virtud del intruso, el dietario de escritor (segunda parte, 2000-2017)", Cuadernos Hispanoamericanos, n 811 (2018), p. 36 : « Hace mucho tiempo, contra lo que algunos dicen creer, que la distinción entre el diario íntimo o personal y el dietario, o diario de escritor, está establecida como orientación sobre la naturaleza de la experiencia literaria que ofrece el autor ».

9. Cette adhésion est claire dans les articles consacrés par Ana Calvo Revilla aux dietarios de Jiménez Lozano en soulignant, notamment, les relations et affinités intellectuelles entre l'écrivain castillan et les auteurs mentionnés dans ses textes. On peut consulter, en particulier, Ana CALVO REVILLA, « Retórica política, cultura y poder. Disputas y controversias en Los cuadernos de Rembrandt de José Jiménez Lozano ", Emilio del Río Sanz, María del Carmen Ruiz de la Cierva et Tomás Albaladejo (éd.), Retórica y Política. Los discursos de la construcción de la sociedad, Logroño, Instituto de Estudios Riojanos, 2012, p. 573-589.

10. Rappelons que Jiménez Lozano a obtenu de nombreux prix littéraires de premier plan en Espagne et, en particulier, le prestigieux Premio Cervantes en 2002.

11. José JimÉnEZ LoZANo, Los cuadernos de Rembrandt, Valencia, Pre-Textos, 2010, p. 36.

12. Ibid., p. 64.

13. Voir Yannick LLORED, «La pensée de Blaise Pascal et de Port-Royal dans l'écriture de José Jiménez Lozano ", Nicole Fourtané et Michèle Guiraud (dir.), Emprunts et transferts culturels dans le monde luso-hispanophone : réalités et représentations, Nancy, Presses Universitaires de Nancy, 2011, p. 189-205.

14. Dans de nombreux textes, l'auteur utilise en effet le terme de « escribidor " pour concevoir sa propre tâche d'écrivain comme on peut le constater, entre autres, dans son discours de réception du Prix Cervantès, en avril 2003, qui se trouve reproduit dans le recueil d'essais : José JIMÉNEZ LOZANO, Siete parlamentos en voz baja, Almería, Confluencias, 2015, p. 137-149.

15. Un des principes de sa morale d'écrivain est défini par Jiménez Lozano à travers l'expression commune "como mejor se puede", laquelle met en lumière l'humilité de l'auteur face à l'expérience créatrice toujours infinie et incommensurable. À cet égard, voir José JIMÉNEZ LOZANO, Los cuadernos de Rembrandt, op. cit., p. 49.

16. Une convergence apparaît entre cet élément primordial de l'écriture de Jiménez Lozano et certains aspects de la pensée du philosophe espagnol Reyes Mate. Sur ce point, on peut consulter Reyes MATE, Tratado de la injusticia, Barcelona, Anthropos, 2011, p. 165 : « El otro modo de pensar va de oído y tiene por modelo la escucha. Pensar es reaccionar a estímulos exteriores, a preguntas o interpelaciones que vienen de fuera de nosotros. Es el modelo de Jerusalén. Lo importante es el otro; lo que tiene valor estructural es la alteridad ya que nos constituimos gracias a la llamada del otro. A ese movimiento que viene del otro y gracias al cual nos constituimos no sólo en sujetos morales, sino en sujetos sin más, lo llamamos compasión ».

17. Cette réalité spirituelle dans le langage, liée à son ontologie de l'existence humaine, rapproche Jiménez Lozano de la philosophie de Martin Heidegger - assez régulièrement cité dans ses essais - ainsi que de celle de Hans- Georg Gadamer. Par rapport à ce dernier, voir, entre autres, Hans Georg GADAMER, L'Art de comprendre. Écrits II. Herméneutique et champ de l'expérience, Paris, Aubier, 1991, en particulier p. 57-67.

18. José JIMÉNEZ LOZANO, Los cuadernos de Rembrandt, op. cit., p. 221-222.

19. Il est intéressant de relever qu'une mise en question assez similaire de cette esthétique de la prétendue transgression, qui devient une sorte de mode plutôt creuse, est présente dans le 
recueil d'essais de Rafael SÁNCHEZ FERLOSIO, La hija de la guerra y la madre de la patria, Barcelona, Destino, 2002, p. 71-75.

20. José JIMÉNEZ LOZANO, Los cuadernos de Rembrandt, op. cit., p. 21.

21. Ibid., p. 216.

22. La critique du relativisme et celle de la régression acceptée des véritables connaissances sur le passé historique et cultuel s'accompagnent parfois, dans les propos de Jiménez Lozano, de la dénonciation de l'idolâtrie dont fait l'objet, selon lui, l'État à travers le contrôle qu'il peut exercer sur la vie de l'individu. Voir Ibid, p. 27 : « El Ídolo-Estado está ahí como un gran sátrapa, revestido de oro y seda, y coronado de majestad sacerdotal y regia, y, a excepción de él, todo es relativo. Los todavía muy recientes horrores de los dos grandes totalitarismos del siglo XX ya van siendo achacados a la perversidad moral de las víctimas; en la modernidad ya no hay víctimas ni verdugos ».

23. Voir, entre autres, José JIMÉNEZ LOZANO, Los cementerios civiles y la heterodoxia española, Barcelona, Seix Barral, 2008, où l'écrivain aborde en détail, à travers l'histoire des cimetières civils en Espagne, les luttes acharnées pour la liberté de croyance et de conscience et son implication dans la vie quotidienne de personnes qui furent souvent marginalisées.

24. José JIMÉNEZ LOZANo, Los cuadernos de Rembrandt, op. cit., p. 211.

25. L'œuvre historiographique d'Américo Castro (1885-1972), intellectuel exilé qui occupa des responsabilités assez importantes dans l'Espagne républicaine, a marqué profondément la pensée de José Jiménez Lozano, en particulier sa vision critique du dogmatisme religieux en Espagne, le lien de celui-ci avec le pouvoir politique et l'importance des rapports entre les trois religions dans l'identité culturelle espagnole. Il est très intéressant de lire leur relation épistolaire: Américo CASTRO y José JIMÉNEZ LOZANO, Correspondencia 1967-1972. Introducción, edición crítica y notas de Guadalupe Arbona y Santiago López-Ríos, Madrid, Trotta, 2020, notamment p. 93-111 où Jiménez Lozano souligne amèrement, entre autres éléments, les difficultés d'acceptation et d'adaptation de l'Église catholique espagnole aux conclusions du concile de Vatican II (1962-1965).

26. À ce propos, on peut constater comment la problématique de la mémoire historique a fait l'objet de nombreuses critiques et de mises en question en Espagne, depuis le début des années 2010, en particulier chez certains intellectuels et écrivains de premier plan, tels que Antonio Muñoz Molina et Javier Marías, qui lui reprochent sa dimension partisane et conflictuelle. En outre, le roman important d'Andrés Trapiello, Ayer no más (Barcelona, Destino, 2012) illustre bien la teneur des critiques formulées contre la mémoire historique en mettant en avant la manière dont cette dernière peut s'allier à la revendication d'une forme de supériorité morale dont la portée idéologique accentue les divisions du passé, lesquelles se trouvent alors projetées et instrumentalisées au sein de certains enjeux socio-politiques du présent historique.

27. Ibid., p. 142.

28. Ibid., p. 143.

29. Ibid, p. 131.

30. Ibid., p. 143.

31. Concernant ce versant significatif de la production littéraire de l'écrivain, on se reportera à ses essais majeurs que sont: José JIMÉNEZ LOZANo, Guía espiritual de Castilla, Valladolid, Ámbito, 2004, et Sobre Judíos, moriscos y conversos. Convivencia y ruptura de las tres castas, Valladolid, Ámbito, 2002. Voir aussi le roman de José JIMÉNEZ LozANo, El mudejarillo, Barcelona, Anthropos, 1992, où se déploie une mise en fiction de certains aspects-clés de la vie du grand poète mystique Jean de la Croix en rapport avec le contexte culturel mudéjar.

32. José JIMÉNEZ LOZANO, Los cuadernos de Rembrandt, op. cit., p. 159.

33. Cette vérité est essentiellement fondée sur les certitudes de l'écrivain qui sont, à un certain niveau, inséparables de sa foi et de ses croyances, mais elle est aussi rattachée à sa volonté de présenter un point de vue critique éclairé, mis en perspective et argumenté, qui montre son 
attachement à une pensée inhérente à une raison délibérative très éloignée des relativismes de la postmodernité et des discours actuels relatifs à la supposée " post-vérité ».

34. José JIMÉNEZ LOZANO, Los cuadernos de Rembrandt, op. cit., p. 46.

\section{RÉSUMÉS}

Cet article propose une analyse de certaines thématiques-clés abordées par l'écrivain José Jiménez Lozano (Langa, Ávila,1930-Valladolid, 2020) dans son dietario - ou cahier de notes personnelles - intitulé Los cuadernos de Rembrandt (2010), telles que la conception de l'écriture et la poétique des textes, mais aussi sa vision critique de la société espagnole du début du XXI ${ }^{\mathrm{e}}$ siècle et de certaines problématiques collectives d'ordre sociopolitique. Tout en éclairant les procédés de composition de l'ouvrage, l'objectif consiste à montrer les liens intimes entre la conception ontologique de l'existence humaine, indissociable de la croyance chrétienne, qui est affirmée par Jiménez Lozano, et sa visée de résistance face aux processus de déshumanisation et de perte des valeurs traditionnelles qui marquent, pour l'auteur, en partie l'époque contemporaine.

This article provides an analysis of certain key themes addressed by José Jiménez Lozano (Langa, Ávila, 1930-Valladolid, 2020) in his dietario - or personal notebook - entitled Los cuadernos de Rembrandt (2010), like the conception of writing and the poetry of the texts but also his critical view of the Spanish society in the early $21^{\text {st }}$ century and about certain sociopolitical issues. While shedding light on the composition methods of this work, the purpose will firstly consist of highlighting the intimate links, affirmed by Jiménez Lozano, between two inseparable themes: the ontological conception of human existence and the Christian belief. Then, the aim will also consist to raise the will for resistance found in his work against the dehumanizing process and the loss of traditional values affecting partially, according to the writer, the contemporary era.

\section{INDEX}

Mots-clés : José Jiménez Lozano, dietario, écriture, critique, croyance, existence

Keywords : José Jiménez Lozano, dietario, writing, critical, belief, existence

\section{AUTEUR}

\section{YANNICK LLORED}

Université de Lorraine, Nancy 